\title{
The slow X-ray pulsar SXP 1062 and associated supernova remnant in the Wing of the Small Magellanic Cloud
}

L. M. Oskinova ${ }^{1}$, M. A. Guerrero ${ }^{2}$, V. Hénault-Brunet ${ }^{3}$, W. Sun ${ }^{4}$, Y.-H. Chu ${ }^{5}$, C. Evans ${ }^{3}$, J. S. Gallagher, III $^{6}$, R. A. Gruendl ${ }^{5}$ and J. Reyes-Iturbide ${ }^{7}$

${ }^{1}$ Institute for Physics and Astronomy, University of Potsdam, 14476 Potsdam, Germany, email: lida@astro.physik.uni-potsdam.de

${ }^{2}$ Instituto de Astrofisica de Andalucia, IAA-CSIC, Granada, Spain

${ }^{3}$ Scottish Universities Physics Alliance (SUPA), Institute for Astronomy, University of Edinburgh, UK

${ }^{4}$ Department of Astronomy, Nanjing University, Nanjing, 210093 Jiangsu, China ${ }^{5}$ Department of Astronomy, University of Illinois, 1002 West Green St., Urbana, IL 61801, USA

${ }^{6}$ Department of Astronomy, University of Wisconsin-Madison, Madison, WI 53706, USA

${ }^{7}$ Escuela Superior de Fisica y Matematicas, IPN, U.P. Adolfo López Mateos, C.P. 07738 D.F., Mexico

\begin{abstract}
SXP 1062 is an exceptional case of a young neutron star in a wind-fed high-mass $\mathrm{X}$-ray binary associated with a supernova remnant. A unique combination of measured spin period, its derivative, luminosity and young age makes this source a key probe for the physics of accretion and neutron star evolution. Theoretical models proposed to explain the properties of SXP 1062 shall be tested with new data.
\end{abstract}

Keywords. stars: emission-line, Be, X-rays: binaries, pulsars: individual (SXP 1062)

\section{Introduction}

Neutron stars are the end products of massive-star's evolution. Stars with an initial mass in excess of $\sim 8 M_{\odot}$ end their life in a core-collapse supernova (SN) explosion giving birth to a degenerate compact object - a neutron star (NS) or a black hole. While the majority of massive stars are born in binary systems, only a small fraction $(\sim 10 \%)$ of binaries survive the SN explosion, leaving a normal star and a compact object in a binary (e.g. Iben \& Tutukov 1996; Popov \& Prokhorov 2006). At some point in binary evolution, the compact object will accrete matter from its companion entering the highmass X-ray binary (HMXB) stage. The detection of pulsations from an accreting X-ray source provides strong evidence that the compact object is a NS. The majority of HMXBs consist of a NS and a Be-type star; these objects are called BeXB (see recent review by Reig 2011). The Be star wind and disk feed the NS making it an X-ray pulsar.

Pulsar spin periods in the range 1-1000s can be explained according to the current understanding of the NS spin evolution (Reig 2011). The spin evolution can be divided into three key phases, characterized by a different energy release mechanism. These phases are known as the pulsar phase, the propeller phase, and the accretor phase. The spin period $P_{\mathrm{eq}}$ is reached when the centrifugal and gravity forces balance. In principle, $P_{\mathrm{eq}}$ is the maximum spin period for a given mass-accretion rate. The mass-loss rates from OB-type stars are reasonably well known, despite the fact that the stellar winds have inhomogeneous structures (Oskinova et al. 2007, Šurlan et al. 2012). 


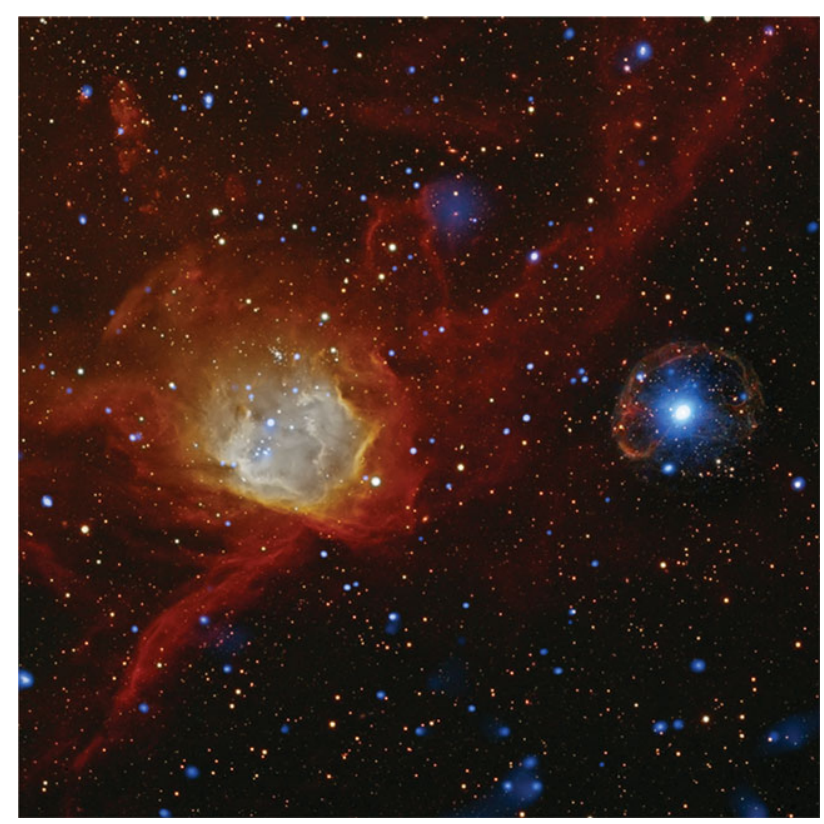

Figure 1. Combined X-ray (blue) and optical (red, yellow) image of NGC 602 and the SNR around the X-ray pulsar SXP 1062. North is up, east is to the left. Image size is $14^{\prime}$, see http://chandra.harvard.edu/photo/2011/sxp1062/

In this canonical model, long periods in excess of 1000 s can be achieved if $B>10^{14} \mathrm{G}$ or $\dot{M}<10^{12} \mathrm{~g} \mathrm{~s}^{-1}$. On the other hand, a new model for wind accretion (Shakura et al. 2012) allows long spin periods and high period derivatives even for standard magnetic fields. The model predicts specific correlations between the behavior of the spin and luminosity. An alternative model to explain long-period pulsars with $P_{\text {spin }} \gg P_{\text {eq }}$ comes from Ikhsanov (2007), who postulates that prior to the accretion powered phase, a subsonic propeller phase may take place. Steady accretion under the condition $P_{\text {spin }}>P_{\text {eq }}$ can be realized when the cooling of the envelope plasma dominates the energy input.

\section{SXP 1062}

Until recently no source was known that allows direct study of various aspects of the theory. The situation changed dramatically with the discovery of the BeXB SXP 1062 (Hénault-Brunet et al. 2012). It was discovered during XMM-Newton and Chandra observations in April-March 2010. SXP 1062 is the first HMXB pulsar firmly associated with a supernova remnant (SNR, see Fig. 1). The SNR SXP 1062 was discovered in $\mathrm{H} \alpha$ and [OIII] filter images (Hénault-Brunet et al. 2012). The Chandra and XMM-Newton $\mathrm{X}$-ray images show that the SNR is filled with X-rays.

The key observational parameters of SXP 1062 were obtained (X-ray pulse period $P=$ $1062 \mathrm{~s}$ and X-ray spectrum) using both Chandra and XMM-Newton observations. Based on the XMM-Newton observations, Haberl et al. (2012) established the spin-down rate of SXP 1062 as $\dot{P} \approx 100 \mathrm{~s} \mathrm{yr}^{-1}$. The association of the pulsar with a SNR constrains the age of the accreting NS to 10-40 kyr. Such young long period pulsars are theoretically not expected.

Figure 1 shows the location of SXP 1062 relative to the young star forming region NGC 602. Some of the most massive stars in the SMC are identified in this cluster (Evans 


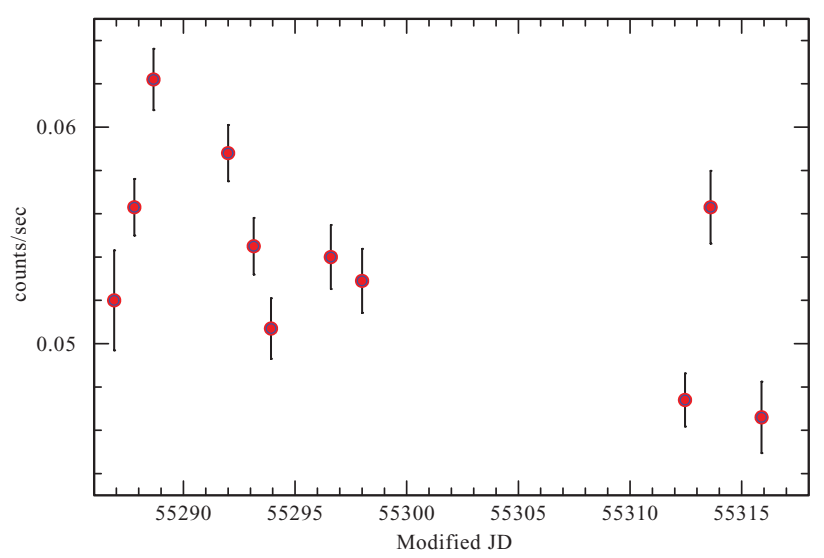

Figure 2. The Chandra ACIS-I light-curve of SXP 1062 in $0.4-10.0 \mathrm{keV}$ band

et al. 2012). The spectral type of the optical companion was confirmed spectroscopically as B0IIIe star. Interestingly, some "normal" B-type stars have strong magnetic fields (Oskinova et al. 2011, and ref. therein), therefore an accretion of a magnetized stellar wind cannot be excluded. There are indications that SXP 1062 has long term X-ray variability. Figure 2 shows the X-ray light curve of SXP 1062 based on 11 Chandra exposures obtained in 2010 .

Presently, four different explanations for the nature of SXP 1062 have been proposed. (1) Haberl et al. (2012) suggested that the NS in SXP 1062 might have been born rotating unusually slowly. If this is the case, no field decay is required. (2) Popov \& Turola (2012) suggested that the NS was born as a magnetar, with an initial magnetic field $B>10^{14} \mathrm{G}$. The strong magnetic braking and field dissipation led to the low $P$ and high $\dot{P}$ as observed. (3) Ikhsanov (2012) proposed that the accretion of magnetized matter can lead to the observed low $P$, while the initial and the current magnetic field strength does not exceed $6 \times 10^{13} \mathrm{G}$. (4) Fu \& Li (2012) suggested that SXP 1062 may be an accreting magnetar, with a present-day field $B>10^{14} \mathrm{G}$. New observations are needed to discriminate among these models and establish the true nature of SXP 1062.

\section{References}

Davies, R. E. \& Pringle, J. E. 1981, MNRAS, 196, 209

Evans, C. J. et al., 2012, ApJ, 753, 173

Iben, I. Jr. \& Tutukov, A. 1996, ApJ, 456738

Ikhsanov, N. R. 2007, MNRAS, 375, 698

Ikhsanov, N. R. 2012, MNRAS, 424, 39

Fu, L. \& Li, X.-D. 2012, ApJ 757, 171

Haberl, F. et al. 2012, A\& $A, 537,1$

Hénault-Brunet, V. et al. 2012, MNRAS, 420, 13

Oskinova, L. M., et al. 2011, MNRAS, 416, 1456

Oskinova, L. M., Hamann, W.-R., Feldmeier, A. 2007,A\& A, 476, 1331

Popov, S. B. \& Prokhorov, M. E. 2006, MNRAS, 367, 732

Popov, S. B. \& Turola, R. 2012, MNRAS, 421, 127

Reig, P., 2011, Ap\&SS, 332, 1

Shakura, N. et al. 2012, MNRAS, 420, 216

Šurlan, B. et al. 2012, A\&A 541, 37 